\title{
AN ABSOLUTE CONTINUITY FOR POSITIVE OPERATORS ON BANACH LATTICES
}

\section{W. FELDMAN and C. PISTON}

\author{
William A. Feldman \\ Department of Mathematics \\ University of Arkansas \\ Fayetteville, Arkansas 72701 \\ CALVIN E. PISTON \\ Department of Mathematics \\ John Brown University \\ Siloam Springs, Arkansas 72761 \\ (Received Ju1y 19, 1990)
}

\begin{abstract}
For positive operators on a Banach lattice, absolute continuity conditions are considered. An operator absolutely continuous with respect to $T$ is compared to sums of compositions of $T$ together with orthomorphisms or in special cases projections. Consequences for compact operators on functions spaces $C(X)$ are considered.
\end{abstract}

KEY WORDS AND PHRASES. Banach lattices, orthomorphisms, quasi-interior points.

1980 AMS SUBJECT CLASSIFICATION CODES. 46B30, $47 B 05,47855$.

\section{INTRODUCTION}

For positive operators $S$ and $T$ between real Banach lattices several types of "absolute continuity" have been defined. Here, we consider an absolute continuity which will be applicable to spaces which are not necessarily Dedekind complete. Several approximations of an operator absolutely continuous with respect to $T$ are provided in terms of sums of operators of the form $Q_{i}{ }^{O T O H} H_{i}$ where $Q_{i}$ and $H_{i}$ are orthomorphisms. These approximations are compared to results known for operators $S$ less than $T$ and for operators on Dedekind complete Banach lattices. We also examine the relationship between this and previous notions of absolute continuity. We begin by recalling the following definitions.

DEFINITION. (Luxemburg [1]). Let E, F be Riesz spaces with S, T positive operators from $E$ to $F$. We say that $\underline{S}$ is absolutely continuous with respect to I if for each positive element $f$ in $E$, we have that $S f$ is in the band generated by Tf. 
DEFINITION. (Feldman [2]). Let E, F be Banach lattices with S, T positive operators from $E$ to $F$. We say that $\underline{S} \underline{i s} \underline{\tau \text {-absolutely continuous }}$ with respect to $I$ if for each positive element $f$ in $E$, we have that $S f$ is in the closure of the order ideal generated by Tf.

We note that for 1 inear functionals on $E=C(X)$ (the continuous real-valued functions on a compact topological space $X$ ) when $E$ is Dedekind complete, these two notions are equivalent to the usual definition of absolute continuity. The absolute continuity introduced here will be shown to be equivalent to the usual notion for functionals on any $C(X)$.

In what follows we will refer to a decreasing sequence $\left[f_{k}\right\}$ of positive elements of a Banach lattice $E$ as a positive decreasing sequence in $\underline{E}$. We now introduce our version of absolute continuity.

DEFINITION. Let $E, F$ be Banach Lattices and let $5, T$ be positive operators from $E$ to $F$. We say that $\underline{S} \underline{\text { is sequentially absolutely continuious }}$ (s-absolutely continuous) with respect to I if for each positive decreasing sequence $\left\{f_{k}\right\}$ in $E$ and each positive linear functional $\phi$ on $F$, we have that $\lim \left(\phi\left(T f_{n}\right)\right)=0$ implies $\lim \left(\phi\left(S f_{n}\right)\right)=0$.

We will be concerned with Banach lattices with quasi-interior points. An element $e$ of a Banach lattice $E$ is a quasi-interior point if the order ideal generated by $e$ is dense in $E$. Recall that the order ideal generated by $e$ is the set of all elements whose absolute value is bounded by some multiple of e. If $E$ is equal to the order ideal generated by an element $e$ then $e$ is an order unit. Recall that if $E$ is a Banach lattice with quasi-interior point, the elements of $E$ can be represented as extended real valued functions on a compact set $X$ each finite on a dense subset (see [3]). We shall call $X$ a representation space for $E$. Further, this representation contains $C(X)$ as a dense order ideal. If $E$ has an order unit, the representation is equal to $C(x)$. We denote by $T$ the subset of the 1 inear operators from $E$ to $F$ which consists of all those positive operators $S$, for which $S$ is s-absolutely continous with respect to $T$. Further, we denote the order ideal generated by a positive operator $T$ by $\langle T\rangle$ and the set of positive operators which are less than some multiple of $T$ by $\langle T\rangle^{+}$. In what follows, we identify elements in a Banach lattice with a quasi-interior point with their representation as extended real valued functions. If $S$ and $T$ are positive operators from a Banach lattice $E$ with a quasi-interior point e to a Banach lattice $F$, the range of $S$ and $T$ is contained in the closure of the lattice generated by the supremum of Se and Te. $X$ will denote the representation space for $E$ and $Y$ the representation space for the Banach lattice generated by the supremum of Se and Te.

\section{ABSOLUTE CONTINUITY.}

We begin with 2 elementary lemmas.

LEMMA 1. Let $S$ and $T$ be 1 inear functionals on $C(X)$, the set of continuous real valued functions on a compact Hausdorff space $X$. Then $S$ is s-absolutely continous with respect to $T$ if and only if the measure associated with $S$ is absolutely continous with respect to the measure associated with $T$.

PROOF. We note that a linear functional $\phi$ on $R$ corresponds to multiplication, thus $\phi\left(T f_{n}\right)$ converges to 0 if and only if $T f_{n}$ converges to 0 . 
It is an easy exercise to see that $S$ is s-absolutely continous to $T$ then the measure associated with $S$ is absolutely continous with respect to the measure associated with $T$.

The converse is a simple application of the Radon-Nikodym Theorem.

LEMMA 2. Let $E$ be a Banach lattice with quasi-interior point $e$ and let $\phi$ be a positive linear functional on $E$. Given a representation space $X$ for $E$, there exists a measure $u$ such that for each $g$ in $E$,

$$
\phi(g)=\int g d u_{\phi}
$$

PROOF. Since $C(X)$ is dense in $E$ and the sequence [g^ne\} converges in norm to $g$ for $g$ non-negative, the sequence $\{\phi(g \wedge n e)\}$ converges to $\phi(g)$. It can be verified that the measure corresponding to the restriction of $\phi$ to $C(X)$ represents $\phi$.

We now give a sufficient condition for s-absolute continuity.

PROPOSITION 1. Let $E$ be a Banach lattice with quasi-interior point $e$ and $F$ a Banach lattice with S, $T$ positive operators from $E$ to $F$. If for each positive decreasing sequence of functions $\left\{f_{n}\right\}$ in $E$ and for each $y$ in the representation space $Y$, the convergence of $T f_{n}(y)$ to 0 implies the convergence of $S f_{n}(y)$ to 0 then $S$ is s-absolutely continous with respect to $T$.

PROOF. Let $\phi$ be a linear functional on $F$ and $\left\{f_{n}\right\}$ be positive decreasing sequence such that $\phi\left(T f_{n}\right)$ converges to 0 . By lemma 2 , we have a measure $u$ such that $\phi(g)=\int g d u$. In particular we have that $\int T f_{n} d u$ converges to 0 . For each $y$ in $Y$, define $h(y)$ by

$$
h(y)=\inf \left\{T f_{n}(y)\right\} .
$$

Thus shdu $\leq \int T f_{n}$ du for each $n$ or shdu $=0$. Setting $A=\{y \mid h(y)=0\}$, we have $u(Y \backslash A)=0$ and $\int_{Y} s f_{n} d u=\int_{A} S f_{n} d u$. Since $h(y)=0$ on $A$ and $\left\{T f_{n}(y)\right\}$ is decreasing, we have that $\operatorname{Tf}_{n}(y)$ converges to 0 on $A$ and thus $S f_{n}(y)$ converges to 0 by hypothesis. Since $S f_{n}(y) \leq S f_{1}(y)$ and $S f_{n}(y)$ converges to 0 on $A$, the Monotone Convergence theorem implies

$$
\lim \int_{Y} S f_{n} d u=\lim \int_{A} S f_{n} d u=0
$$

Thus we have that $\phi\left(S f_{n}\right)$ converges to 0 , that is that $S$ is s-absolutely continous with respect to $T$.

We note that in the case when $F=C(Y)$, the converse of the proposition is also true since yoT defines a positive linear functional on $F$.

It is obvious that if $S<T$ then $S$ is s-absolutely continous with respect to $T$, i.e. contains $\langle T\rangle^{+}$. It is an easy exercise to show that $\tau$ is closed and thus contains even the closure of $\langle T\rangle^{+}$.

PROPOSITION 2. $\dddot{T}$ is a closed subset of $L(E, F)$, the 1 inear operators from $E$ to $F$ with respect to the operator norm. In particular, $\tau$ contains the closure of $\langle T\rangle^{+}$

We now compare and contrast these notions of order and absolute continuity when the range is an M-space. 
THEOREM 1. Let $E$ be a Banach lattice with quasi-interior point and $S$, $T$ be positive operators from $E$ to $C(Y)$. Consider the conditions.

i) $S$ is $\tau$-absolutely continous with respect to $T$ (in the sense of Luxemburg)

ii) $S$ is $\tau$-absolutely continous $w$ ith respect to $T$

iii) $S$ is s-absolutely continous with respect to $T$

iv) $S$ is in the closure of $\langle T\rangle^{+}$.

Then we have

$$
i v \Rightarrow i i i \Rightarrow i i \Rightarrow i
$$

and no other implications hold.

PROOF. That ( $i v$ ) implies $(i j i)$ is Proposition 2. To show that ( $i i i)$ implies ( $i$ i) we first note that if $S$ is s-absolutely continous with respect to $T$ and $f \geq 0, S f(y)>0$ implies $T f(y)>0$. For a given $\varepsilon>0$ and $g$ such that $0<g \leq S f$, let $A$ be the set $\{y \mid(g-\varepsilon e)(y) \geq 0\}$. Then $A$ is compact and hence there exists a $\lambda>0$ such that $\operatorname{Tf} \geq \lambda(g-\varepsilon e)$. Therefore we have that $T f \geq \lambda(S f-\varepsilon e)$ and thus $(S f-\varepsilon e) V O$ is in the order ideal generated by Tf and therefore $S F$ is in the uniform closure of the ideal generated by Tf. Thus we have ( $i$ i i) implies ( $i i)$. That ( $i i$ ) implies (i) follows from the fact that the closure of the ideal generated by $T f$ is contained in the band generated by Tf.

That no other implications hold is shown by the following examples. We will assume that $C(X)$ is endowed with the sup-norm topology.

EXAMPLE 1. We give here operators $S$ and $T$ such that $S$ is absolutely continuous with respect to $T$, but $S$ is not $\tau$-absolutely continuous with respect to $T$.

Define $S$ and $T$ from $C[0,1]$ to $C[0,1]$ as follows.

$$
\begin{aligned}
& S f(x)=f(x) \\
& T f(x)=x f(x)
\end{aligned}
$$

Sf is in the band generated by $T f$ and hence $S$ is absolutely continous with respect to $T$.

However, for each operator $T^{\prime}$ such that $T^{\prime} f$ is in $\langle T f\rangle$, we have for any positive $f$ in $C(X), T^{\prime} f(0)<\lambda T f(0)=0$, for some $\lambda$ in $R^{+}$, and thus $T^{\prime} f(0)=0$. Therefore,

$$
|| S f-T^{\prime} f|| \geq\left|S f(0)-T^{\prime} f(0)\right|=|S f(0)|
$$

If $f(0) \neq 0$ then $5 f$ is not in the closure of the order ideal generated by Tf and thus $S$ is not $t$-absolutely continuous with respect to $T$.

EXAMPLE 2. Here we give operators $S$ and $T$ such that $S$ is $\tau$-absolutely continous with respect to $T$, but $S$ is not $s$-absolutely continous with respect to $\mathrm{T}$.

Let $N^{*}$ denote the one point compactification of $N$. Define operators from $C\left(N^{*}\right)$ to $C\left(N^{*}\right)$ as follows. 


$$
\begin{aligned}
& S f(x)=f(x) \\
& T f(x)=\left\{\sum_{n=1}^{\infty}[f(n)] /\left(n^{2}\right)\right\} 1 \quad(1 \text { denotes } f(x)=1)
\end{aligned}
$$

Since Tf is constant, $S f$ is less than some multiple of Tf, i.e. in the order ideal generated by Tf. Thus $S$ is t-absolutely continous with respect to $T$. However, by defining $f_{k}(x)$ on $N$ by

$$
f_{k}(x)=x_{[k, \infty]}
$$

we have a positive decreasing sequence of functions on $C\left(N^{*}\right)$ with $\operatorname{Tf}_{k}(x)$ converging to 0 for each $x$ in $N^{*}$. However, $S f_{k}(\infty)=1$ for each $k$, and hence does not converge to 0 . Thus $S$ is not s-absolutely continous with respect to T.

EXAMPLE 3. Here we give operators $S$ and $T$ such that $S$ is $s$-absolutely continous with respect to $T$, but where $S$ is not in the closure $\langle T\rangle^{+}$. We define operators from $C\left(N^{*}\right)$ to $C\left(N^{*}\right)$ as follows,

$$
\begin{aligned}
& S f=f \\
& \left.T f=\left[\sum_{n=1}^{\infty}[f(n)] / n^{2}\right]+f(\infty)\right\} 1 .
\end{aligned}
$$

Suppose that $S$ is in the closure of $\langle T\rangle^{+}$. Let $T^{\prime} \varepsilon\langle T\rangle^{+}$. For $x$ in $N^{*}$, we have for some $\lambda$ and for every $f \geq 0$ in $C\left(N^{*}\right)$,

$$
T^{\prime} f(x)<\lambda T f(x)=\lambda\left(\sum_{n=f}^{\infty}[f(n)] / n^{2}+f(\infty)\right)
$$

Pick $m$ such that $m^{2}>2 \lambda$, and define a function $g$ by

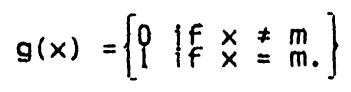

We thus have $g$ in $C\left(N^{*}\right)$ and $\|g\|_{\infty}=1$. In addition, for every $x$ in $N^{*}$,

$$
T^{\prime} g(x)<\lambda\left(1 / m^{2}\right)<1 / 2
$$

Thus,

$$
\begin{array}{rl}
\mid 15-T^{\prime} 11 & 2\left|15 g-T^{\prime} g\right| 1 \\
& 2\left|S g(m)-T^{\prime} g(m)\right|>1 / 2
\end{array}
$$

and thus $S$ can not be in the closure of the order ideal generated by $T$.

It is routine to check using Proposition 1 that $S$ is s-absolutely continous with respect to $T$.

\section{APPROXIMATIONS.}

The next several theorems give approximations which enlarge the scope of previous results. Recall that a positive operator $T$ from a Banach lattice $E$ into itself is an orthomorphism if and only if there exists an element $m$ in $E$ such that $T f=m f$ for each $f$ in $E$ where the elements are viewed in the representation (see e.g. [4], Thm. 4). 
The next theorem is based on a result for operators $S$ which are $\tau$-absolutely continous with respect to $T$ on Dedekind complete M-spaces in [2] using a similar argument.

THEOREM 2. Let $E$ and $F$ be Banach lattices with quasi-interior points and let $S, T$ be positive operators from $E$ to $F$ such that $S$ is $\underline{s}$-absolutely continous with respect to $T$. Then for every $f$ in $E$, and every $\varepsilon>0$, there exist orthomorphisms $H_{i}$ on $E$ and $Q_{i}$ on $F$, for a finite number of indices $i$, such that

$$
\left\|\left(S-\sum_{i=1}^{n} Q_{i} O T O H_{i}\right) f\right\|<\varepsilon,
$$

where $11 \cdot 11$ is the Banach space norm on $F$.

PROOF. If $e$ is a quasi-interior point of $E$ then for a given $f$ in $E^{+}$, we have that $e+f$ is also a quasi-interior point. Thus we can choose the representation space $X$ so that if $f$ is in $C(X)$ and $S f$ and Tf are in $C(Y)$. Now, assume that $S$ is s-absolutely continous with respect to $T$. For each fixed $y$ in $Y$, let $u_{y}$ and $v_{y}$ be the measures corresponding to the functionals (yoT) and (yos), respectively. By the Riesz Representation Theorem,

$$
\begin{aligned}
& \operatorname{Tf}(y)=(y \circ T)(f)=\int f d u_{y} \text { and } \\
& S f(y)=(y \circ S)(f)=\int f d v_{y} .
\end{aligned}
$$

As noted in Lemma $1, v_{y} \ll u_{y}$ and thus we have by the Radon-Nikodym Theorem a positive measurable function $g_{y}$ on $x$ such that

$$
s f(y)=\int f g_{y} d u_{y} .
$$

Since $\left(y_{0} S\right)$ is continuous, we have that $S(1) y<\infty$, and therefore $g_{y}$ is in $L^{1}\left(X, u_{y}\right)$. Given $\varepsilon>0$ and $f$ in $C(X)$, there is an $h_{y}$ in $C(X)$ such that

$$
\left\|g_{y}-h_{y}\right\|_{1}<\varepsilon /\|f\|_{\infty} \quad \text { (see [5], Thm 25.10). }
$$

Thus we have

$$
\begin{aligned}
\left|\left[T\left(h_{y} f\right)-s f\right] y\right| & =\left|\int\left(h_{y}-g_{y}\right) f d u_{y}\right| \\
& <|| h_{y}-\left.g_{y}\left|\|_{1}\right||f|\right|_{\infty}<\varepsilon .
\end{aligned}
$$

Since $T, S, f, h_{y}$ are all continuous, there exists a neighborhood $N_{y}$ of $y$ such that for every $z$ in $\mathrm{N}_{\mathbf{y}}$,

$$
\left|\left[T\left(h_{y} f\right)-S f\right] z\right|<\varepsilon
$$

For each $y$ in $Y$, choose such a neighborhood. Since $Y$ is compact there is a finite number of these neighborhoods which cover $Y$. We label the neighborhoods $N_{i}$ for $i=1,2, \ldots n$. Further, functions $q_{i}$ in $C(Y)$ can be chosen (a partition of unity, see e.g. [6], p. 63) such that $0 \leq a_{i} \leq, 1, \sum_{i=1}^{n} q_{i}=1$, and $a_{i}(y)=0$ on the complement of $N_{i}$. Let $h_{i}=h_{y}$, where $N_{i}=N_{y}$. 
Assume that $z$ is in $Y$ such that $q_{i}(z) \neq 0$. Then $z$ is in $N_{i}$ and

$$
\left|S f(z)-T\left(f h_{i}\right)(z)\right|<\varepsilon
$$

and hence

$$
q_{i}(z)\left|S f(z)-T\left(f h_{i}\right)(z)\right|<\varepsilon q_{i}(z)
$$

Therefore, summing up over the index $i$, we have

$$
\sum_{i=1}^{n}\left(q_{i}(z)\left|S f(z)-T\left(f h_{i}\right)(z)\right|\right)<\varepsilon \sum_{i=1}^{n} q_{i}(z) .
$$

thus,

$$
\left|S f(z)-\sum_{i=1}^{n} q i(z) T\left(f h_{i}\right)(z)\right|<\varepsilon .
$$

Since $z$ is arbitrary, this gives us

$$
\mid 15 f-\sum_{i=1}^{n} q_{i} T\left(f h_{i}\right) \|_{\infty}<\varepsilon .
$$

We define orthomorphisms $Q_{i}$ on $C(Y)$ and $H_{i}$ on $C(X)$ by

$$
\begin{aligned}
& Q_{i} f(y)=q_{i}(y) f(y) \\
& H_{i} f(x)=f(x) h_{i}(x) .
\end{aligned}
$$

Thus we have by extending to $E$ and $F$ (e.g., see [2])

$$
\left\|\left(S-\sum_{i=1}^{n} Q_{i} \circ T O H_{i}\right) f\right\|_{\infty}<\varepsilon .
$$

Hence,

$$
\left|\left(S-\sum_{i=1}^{n} Q_{i}{ }^{\text {OTOH }}{ }_{i}\right) f\right|<\varepsilon \cdot 1 \text {, }
$$

Which implies for the Banach space norm,

$$
\|\left(S-\sum_{i=1}^{n} Q_{i} \text { oT OH } H_{i}\right) f\|<\| \varepsilon \cdot\|\|=\varepsilon \cdot\|e\|==\varepsilon \text {. }
$$

When the spaces involved are Dedekind complete, approximations of this type have generally been given using projection operators (e.g. [7], [8]).

CORROLARY 1. Let $E, F, S, T$ be as in the theorem. If $E$ and $F$ are Dedekind complete, and if $S$ is s-absolutely continous with respect to $T$ then, for each positive $f$ in $E$ and every $\varepsilon>0$, there exist projection operators $Q_{i}$. $H_{i}$ and real valued scalers $\alpha_{i}$ for a finite number of indices $i$ such that

$$
||\left(S-\sum_{i=1}^{n} a_{i} Q_{i} \text { OTOH }{ }_{i}\right) f||<\varepsilon .
$$

PROOF. If $C(X)$ is Dedekind complete then we have that $x$ is extremally disconnected. In this case each simple function of the form $\alpha_{i} x_{\overline{0}}$ is continuous, if $\mathbf{O}_{\mathfrak{i}}$ is open. Thus, following the proof of the Theorem we choose $h_{y}$ to be a simple function of the form $\sum_{i=1}^{n} \alpha_{i} x_{H_{i}}$ with $H_{i}$ open. Further, we choose $q_{i}$ to be characteristic functions of clopen sets. Defining the operators $H_{i}$ and $Q_{j}$ as 


$$
\begin{aligned}
& H_{i} f=f x_{\bar{H}_{i}} \\
& Q_{j} f=q_{j} f
\end{aligned}
$$

we observe that each $Q_{j}$ and $H_{i}$ are projections. The remainder of the proof is similar to that of Theorem 2 .

The next result, motivated by results which were established for operators $S$ which are in the ideal generated by $T$ (on M-spaces by Aliprantis and Burkinshaw [6] and on Banach lattices with quasi-interior points by Haid [9]), is a direct corollary of Theorem 2.

COROLLARY 2. Let $E$ and $F$ be Banach lattices with quasi-interior points and let $S, T$ be positive operators from $E$ to $F$ such that $S$ is $s$-absolutely continous with respect to $T$. Then for every $f$ in $E, \phi$ a positive linear functional on $F$, and $\varepsilon>0$, there exist orthomorphism $H_{i}$ on $E$ and $Q_{i}$ on $F$, for a finite number of indices $i$ such that

$$
\phi\left(\left|\left(S-\sum_{i=1}^{n} Q_{i} \circ T^{\circ} \circ H_{i}\right) f\right|\right)<\varepsilon .
$$

A further characterization of s-absolute continuity is given by the following.

THEOREM 3. Let $E$ and $F$ be Banach lattices with quasi-interior points and let $S$ and $T$ be positive operators from $E$ to $F$. Then $S$ is $s$-absolutely continous with respect to $T$ if and only if, given $\varepsilon>0, f$ in $E$ and $\phi a$ positive linear functional on $F$, there exists an orthomorphism $H$ on $E$ such that $|\phi(T H-5) g|<\varepsilon$ for all $g$ in $E$ with $|g|<f$.

PROOF. Let $S$ be s-absolutey continous with respect to $T$. If $e$ is a quasi-interior point of $E$ then so is $f+e$. We choose a representation space so that $f$ is in $C(X)$. For a linear functional $\phi$ we have that $\phi 0 S \ll$ \&OT as measures on $X$ (see lemna 1 ). Thus if for every $h$ in $C(X)$ we have

$$
\phi \circ T(h)=\text { Shdu }
$$

then there is a positive measurable function $p^{\prime}$ such that

$$
\phi 0 S(h)=\int h p^{\prime} d u
$$

Letting $h(x)=1$ for every $x$, we see that $p^{\prime}$ is $L^{\prime}(u)$, and so there exists a function $p$ in $C(X)$ such that ||$p-p^{\prime}\|\|_{\mid}<\varepsilon /\|f\|_{\infty}$. Therefore for $g$ such that $|g|<f$, we have

$$
\begin{aligned}
& |(\phi \circ S) g-(\phi \circ T p) g|=\left|\int g p^{\prime} d u-\int h g d u\right|=\left|\int g\left(p-p^{\prime}\right) d u\right| \\
& \leq|| p-p^{\prime}||_{1}|| g||_{\infty}<\varepsilon .
\end{aligned}
$$

Let $H$ be the orthomorphism defined by multiplication by $p$. Thus for every $g$ in $E$ with $|g|<f$, we have that

$$
|\phi(T H-S) g|<\varepsilon .
$$

Conversely, let $\left\{f_{n}\right\}$ be a positive decreasing sequence in $E$ such that $\phi\left(T f_{n}\right)$ converges to 0 . Again choosing $X$ so that $f_{1}$ is in $C(X)$, we have $\left\{f_{n}\right\}$ in 
$C(X)$. By assumption, given $\varepsilon>0$, there exists an orthomorphism $H$ on $E$ so that $\left|\phi(T H-S) f_{n}\right|<\varepsilon$ for every $n$. Recall that $H$ is a multiplication operator, say multiplication by $p$ in $C(X)$. Now ( $\phi 0 T$ ) is a positive linear functional on $C(X)$ corresponding to a measure, say $u$. since $\phi\left(T f_{n}\right)=\int f_{n} d u$ converges to 0 , it follows that $\int f_{n} p d u=\phi\left(T H f_{n}\right)$ converges to 0 . Thus $\phi\left(S f_{n}\right)$ also converges to 0 and so $S$ is s-absolutely continous with respect to $T$.

For compact operators on M-spaces, we have the following which is based on a result for Dedekind complete spaces in [2].

THEOREM 4. Let $X, Y$ be compact Hausdorff spaces and $S, T$ be positive operators from $C(X)$ to $C(Y)$. If $S$ is s-absolutely continous with respect to $T$ and $T$ is compact, then $S$ is compact if and only if $S$ is the norm limit of operators of the type $\sum_{i=1}^{n} Q_{i} \circ{ }^{\circ} \circ H_{i}$, for a finite number of indices and each $Q_{i}$ and $H_{i}$ is an orthomorphism.

PROOF. If $T$ is compact then each operator of the form $Q_{i}{ }^{\circ}{ }^{\circ} H_{i}$ is compact and hence so is the finite sum $\sum_{i=1}^{n} Q_{i} \circ T_{0 H}$, and thus $S$ is compact.

For the converse, we assume that $S$ is compact. For every $y$ in $Y$ and $\varepsilon>0$, denote by $G$ the operator defined by

$$
G f=S f-T\left(h_{y} f\right) \text {, }
$$

where $h_{y}$ is the continuous function as described in the proof of Theorem 2. Letting $H_{y}$ be the orthomorphism on $C(X)$ defined by multiplication by $h_{y}$, we have that $G$ is compact, since both $S$ and $T H_{y}$ are compact. Further, as in the proof of Theorem 2, we have

$$
|G f(y)|<\varepsilon \text {. }
$$

We will show that there is a neighborhood $N_{y}$ of $y$ such that $G f\left(N_{y}\right)$ is contained in $(-3 \varepsilon, 3 \varepsilon)$ for all $f$ in $C(X)$ such that ||$f||_{\infty} \leq 1$.

Assume that this is not the case. Then there exists a net $\left\{y_{\alpha}\right\}$ in $Y$ such that $y_{\alpha}$ converges to $y$, and there exist functions $f_{\alpha}$ in $C(x)$ with $\mid\left\|_{\alpha}\right\|_{\infty}$ $\leq 1$ and satisfying both of the following

i) $\mathrm{Gf}_{\alpha}\left(y_{\alpha}\right)>3 \varepsilon$, for all $\alpha$.

11) $G f_{\alpha}(y)<\varepsilon$.

Since $G$ is compact, there exists a subnet of $\left\{\mathrm{GF}_{\alpha}\right.$ \} converging to some function $g^{\prime}$ in $C(Y)$. However, from condition $\left.i i\right)$ we have

$$
g^{\prime}(z)<3 \varepsilon / 2
$$

for all $z$ in some neighborhood $w$ of $y$, while from condition (i) we see that

$$
g^{\prime}\left(y_{\alpha}\right)>3 \varepsilon
$$

for all $y_{\alpha}$ in $W$, giving us a contradiction.

Therefore, there exists a neighborhood $N_{y}$ of $y$ such that $G f\left(N_{y}\right)$ is contained in $(-3 \varepsilon, 3 \varepsilon)$, for all $f$ with $\left.|| f\right|_{\infty} \leq 1$. Repeating the construction 
process of Theorem 2, we find a partition of unity $\left\{a_{i}\right\}$ for a cover $\left\{N_{i}\right\}$ of $Y$ such that

$$
0 \leq a_{i} \leq 1, \sum_{i=1}^{n} a_{i}=1 \text {, and } a_{i}(y)=0 \text { on the complement of } N_{i} \text {. }
$$

Thus we have

$$
|| S-\sum_{i=1}^{n} Q_{i} \circ T \circ H_{i}||=\sup \left\{|| S f-\sum_{i=1}^{n}\left(Q_{i} \circ T \circ H_{i}\right) f\|\|_{\infty}\right\}<3 \varepsilon
$$

and thus $S$ is the norm limit of operators of the type $\sum_{i=1}^{n} Q_{1} \circ \mathrm{OT}_{\mathrm{O}} \mathrm{H}_{i}$.

We further note that the approximation given in Theorem 2 is not, in general, uniform. Let $E=F=C\left(N^{*}\right)$ and define $S f(x)=f(x)$ and $T f(x)=$ $\left.\left(\sum_{n=1}^{\infty} f(n) / n^{2}+f(\infty)\right)\right)$. As stated earlier $S$ is s-absolutely continous with respect to $T$. $T$ is compact (it has rank 1 ), but $S$ is not compact. If the approximation given in Theorem 2 were uniform, then Theorem 4 would imply $S$ is compact.

\section{REFERENCES}

1. Luxemburg, W., Some Aspects of the Theory of Riesz Spaces, Fayetteville, University of Arkansas Press (1979)

2. Feldman, W. "Positive Operators on Banach Lattices and the Radon Nikodym Theorem", Proc. of the AMS, 100 (3), 517-521 (1987)

3. Schaeffer, H., Banach Lattices and Positive Operators, Berlin, Springer Verlag (1974)

4. Feldman, W. and Porter, J., "Operators on Banach Lattices as Weighted Compositions", J. London Math. Soc., 33 (1), 149-156 (1986)

5. Aliprantis, C.D. and Burkinshaw, 0., Principles of Real Analysis, New York, North Holland (1981)

6. Aliprantis, C.D. and Burkinshaw, 0., Positive Operators, New York, Academic Press (1985)

7. Aliprantis, C.D. and Burkinshaw, 0., "The Components of a Positive Operator", Math. Zeit,. 184 (2), 245-258, 1983).

8. dePagter, B., "The Components of a Positive Operator", Indag. Math., 229-241, (1983)

9. Haid, W., "Satze von Radon Nikodym typ fur Operatoren auf Banach Verbunden", Dissertation, Univ. Tubingen (1982)

10. Aliprantis, C.D. and Burkinshaw, 0 . "Positive Compact Operators on Banach Lattices", Math Zeit., 174, 289-298 (1980).

11. Kalton N. and Saab, P., "Ideal Properties of Regular Operators between Banach Lattices", 111.J. of Math., 29 (3), 382-400 (1985) 


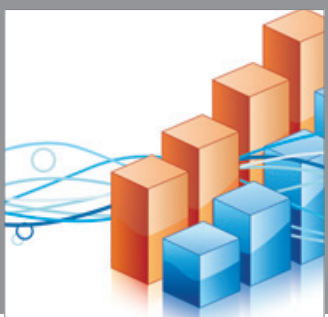

Advances in

Operations Research

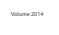

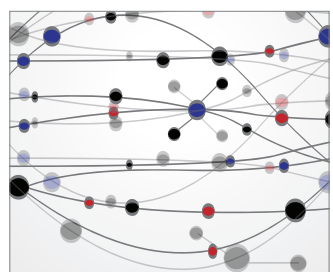

\section{The Scientific} World Journal
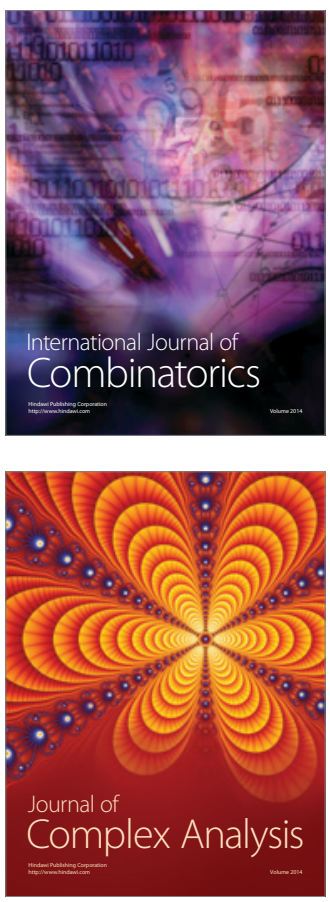

International Journal of

Mathematics and

Mathematical

Sciences
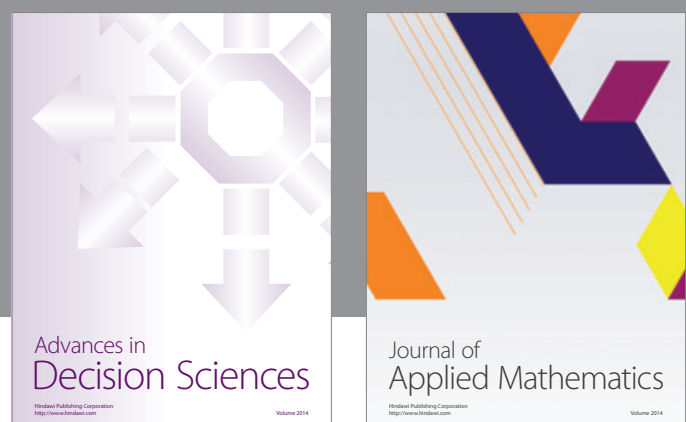

Journal of

Applied Mathematics
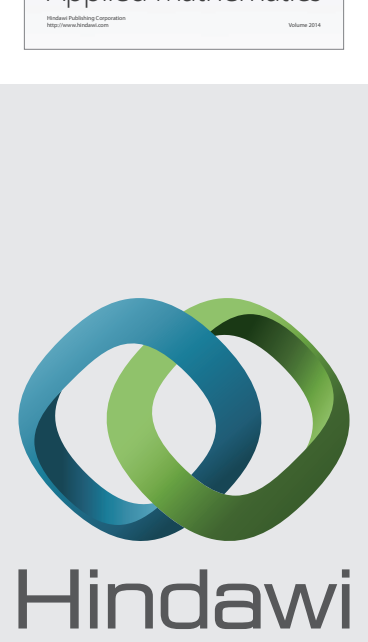

Submit your manuscripts at http://www.hindawi.com
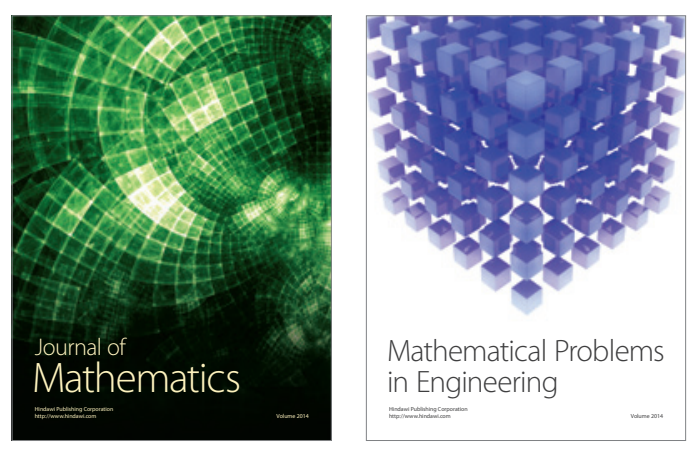

Mathematical Problems in Engineering
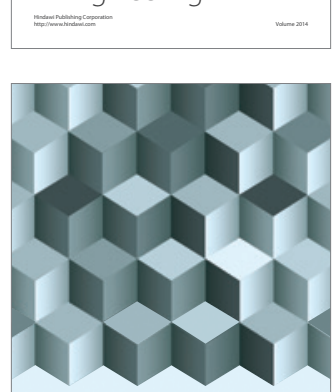

Journal of

Function Spaces
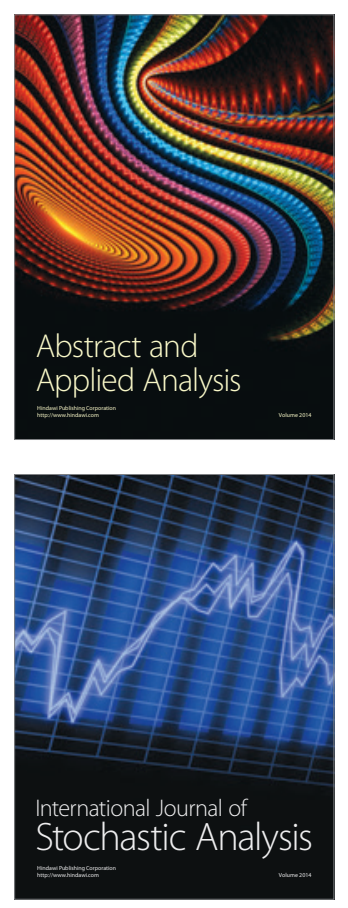

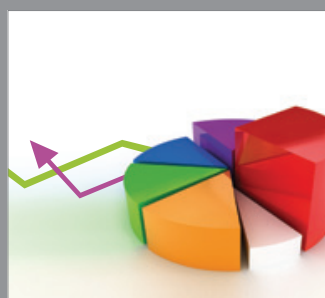

ournal of

Probability and Statistics

Promensencen
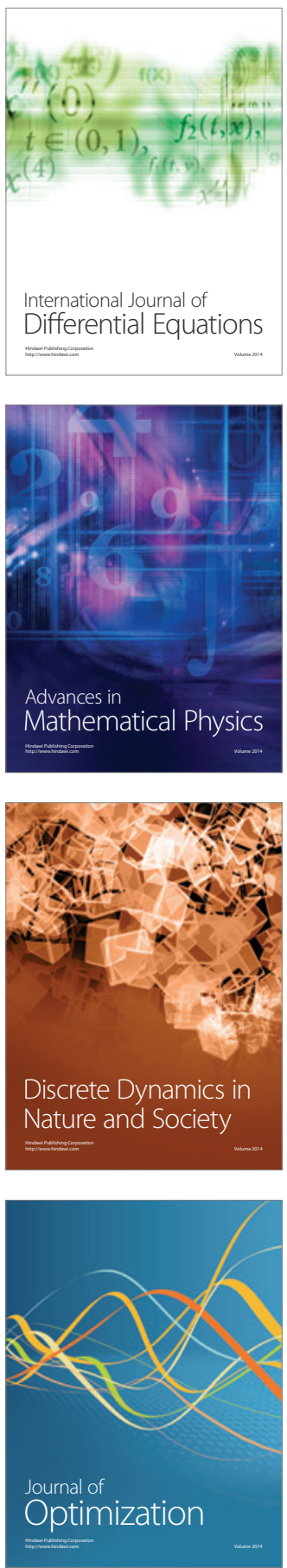